\title{
Digital Acculturation or Displacement?: Examining the Link Between Social Media and Well-Being
}

\author{
Shu-Sha Angie Guan * \\ Department of Child and Adolescent Development, California State University, Northridge, Northridge, CA, United States
}

\section{OPEN ACCESS}

Edited by:

Peter David Tolmie,

University of Siegen, Germany

Reviewed by:

Jessica Dennis,

California State University, Los Angeles, United States

Yalda Uhls,

University of California, Los Angeles,

United States

*Correspondence:

Shu-Sha Angie Guan

angie.guan@csun.edu

Specialty section: This article was submitted to

Digital Impacts,

a section of the journal

Frontiers in Human Dynamics

Received: 21 July 2020

Accepted: 09 June 2021

Published: 01 July 2021

Citation:

Guan S-SA (2021) Digital Acculturation or Displacement?: Examining the Link Between Social Media and Well-Being.

Front. Hum. Dyn 3:585906.

doi: 10.3389/fhumd.2021.585906
The current study applies digital media and acculturation models to assess the links between offline social connection, online social media use, and well-being. Acculturation research suggests that intercultural contact can create conflict that individuals must resolve using four acculturations strategies: marginalization, separation, assimilation, or integration. It suggests that those who fare best adopt strategies in which they maintain heritage connection, identity and values [e.g., offline, face-to-face (F2F) social connection] while also connecting with people and norms in the new context (i.e., online). The social interaction displacement hypothesis suggests that high media use that displaces inperson social interaction (i.e., assimilation strategy) can lead to poor outcomes. The results from a survey of 60 college students ( $M_{\text {age }}=22.02, S D=3.54 ; 73.3 \%$ female) suggest that individuals who maintain high offline, F2F social interaction (i.e., separation strategy) fared better than those who adopted assimilation or even integrations strategies. The findings have implications for people who spend more time online, perhaps at the expense of inperson socialization, and introduce novel opportunities for the understanding of the link between social media and poor mental health outcomes.

Keywords: social media, acculturation, well-being, self-esteem, social support

\section{INTRODUCTION}

In the last 2 decades, mobile and social media have made it easier to "connect" with others. Although this increased capacity for social communication and support may have benefits for psychosocial functioning, particularly for adolescents (Jensen et al., 2019; Orben, 2020), meta-analyses have linked digital media use to lower self-esteem, greater loneliness (Song et al., 2014; Liu and Baumeister, 2016) and depressive symptoms (Yoon et al., 2019). At the societal level, increases in technology have also been linked to increases in depression and suicidality rates (Twenge, 2020). Frameworks that examine the interaction between the two social contexts (i.e., the offline and online) with highly permeable boundaries rather than focusing on either offline contexts or online use solely would shed light on this paradox. Given the cultural nature of each social context and frequent "border-crossings," the current study tests two frameworks in the domains of digital media and acculturation (i.e., the cultural and psychological change in the adaptation from one cultural context to another) to examine how people navigate and engage in relationships offline and online in ways that differentially affect psychological and social well-being: a digital media acculturation model based on Berry's (2005) cultural psychological work and displacement of in-person social interaction, a mechanism proposed by Twenge (2020) to explain how digital media use may affect mental health.

Berry (2005) hypothesized that intercultural contact, that occurs in "cultural migration" between physical and geopolitical spaces, creates internal conflict between two major issues that people must 


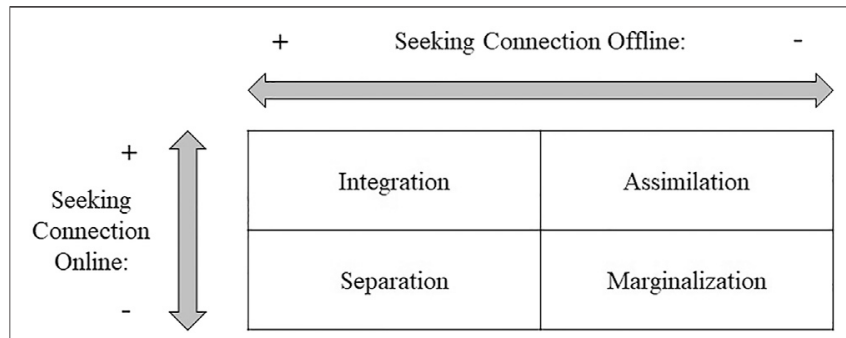

FIGURE 1 | Digital Acculturation Model and Strategies.

reconcile: 1) their orientation or "a relative preference for maintaining one's heritage culture and identity, and 2) a relative preference for having contact with and participating in" the host society (Berry, 2005, p. 704). Their adaptive strategy is derived from reconciliation of these two issues and results in four types of acculturation strategies: marginalization (cultural loss and exclusion from the new social context), separation (cultural maintenance but avoidance of interaction with new social norms), assimilation (cultural loss that coincides with seeking interaction with another culture), and integration (cultural continuity while also incorporating new social structures and interactions with people in the new context).

The same can be said for movement into digital and virtual social spaces. In Figure 1, I have reimagined the acculturation strategies in the cultural migration from the offline to online digital media contexts. The focus in the current study is on social media rather than other technology use because culture is socially constructed and, therefore, inherently social. Additionally, social experiences have important implications for mental and physical well-being (e.g., Uchino, 2006). The idea of "cultural migration" from offline to online contexts is what spurred education writer, Marc Prensky (2001), to coin the phrase "digital natives" to describe recent generations of youth who have grown up fully immersed in digital media (or at least, who are more frequent sojourners) relative to previous generations of "digital immigrants." For example, there is evidence that "digital natives," who have presumably assimilated to online contexts, experience social connection differently than their "digital immigrant" counterparts (Chan, 2015).

In the acculturation literature, pursuing an integration strategy has been associated with the most positive outcomes (e.g., lower acculturative stress, higher self-esteem and sense of well-being; Berry, 2017). There is also evidence to suggest that maintaining ties to heritage culture in general (i.e., adopting integration or separation strategies), perhaps due to maintained access to economic and social resources during the potentially-fraught adaptation process, may promote mental health relative to adopting an assimilation or marginalization strategy (Berry and Hou, 2016). Therefore, a digital media acculturation model would lead to the following hypotheses:

$\mathrm{H1}$ : Broadly, people who maintain high offline social connection (i.e., use an integration or separation strategy) will report greater psychological well-being than those who report low offline social connection (i.e., use a marginalization or assimilation strategy), though those who relate to both online and offline communities (i.e., integration) will fare best psychologically.

$\mathrm{H} 2$ : People who maintain high offline social connection (i.e., use an integration or separation strategy) will report greater social well-being than those who report low offline social connection (i.e., use a marginalization or assimilation strategy), though those who relate to both online and offline communities (i.e., integration) will fare best socially.

In line with acculturation literature, digital media research suggests that both complete exclusion from online social communication among nonusers as well as high social media use (e.g., greater than $2 \mathrm{~h}$ a day), perhaps at the expense of inperson social interaction, can be risk factors for poorer mental health (Przybylski et al., 2020; Twenge, 2020). That is, high digital media use that displaces time away from face-to-face (F2F) social interaction might explain the link between digital media use and mental health. Additionally, the greater use of digital devices and modalities for social communication has been associated with poorer psychological well-being (e.g., life meaning, relationship quality), particularly in the face of weaker in-person connections for young adults age 18-34 (Chan, 2015). If the social displacement hypothesis is true, the following will be supported:

H3: People who report high online communication but low offline communication (i.e., adopt an assimilation strategy) will have the poorest psychological and social well-being outcomes.

The current study expands on the existing digital media literature as well as incorporates perspectives from cultural psychology in ways that help explain how changing social media landscapes and people's responses to them can affect individual health and development. These frameworks are not necessarily at odds. Testing both can help identify the conditions in which cultural adaptation and negotiation between offline and online social contexts shape people's experiences and sense of well-being.

\section{MATERIALS AND METHODS}

\section{Participants and Procedure}

College students $\left(N=60 ; M_{\text {age }}=22.02, S D=3.54 ; 73.3 \%\right.$ female $)$ from diverse backgrounds (e.g., 5\% African American, $26.7 \%$ Asian American, 43.3\% Latino, 10\% White or Caucasian, $11.7 \%$ multi-ethnic, and 3.3\% other ethnicity such as Middle Eastern) were recruited via campus fliers, class presentations, and emails in 2017. Average mother's education and father's education was between technical/trade school and community college $(M s=$ $3.32,3.33, S D s=1.54,1.67$ on a scale from $0=$ no formal education to $6=$ graduate/law/medical school) and average years in the United States was $18.63(S D=6.14)$.

\section{Measures}

Social Media Use. Issue 1 (Figure 1: maintaining ties offline) was assessed using a frequency of in-person social connection (i.e., "how much time do you spend having face-to-face conversations?") and Issue 2 (Figure 1: preference for having contact with and participating in the host online society or environment) was assessed using a frequency of social media use item (i.e., "how much time do you spend emailing, sending messages, and posting on 
social media?") on a scale from $1=$ less than $1 \mathrm{~h} /$ day, $2=$ about $1-2 \mathrm{~h} /$ day, $3=$ about $2-3 \mathrm{~h} /$ day, $4=$ about $3-4 \mathrm{~h} /$ day , and $5=$ more than $4 \mathrm{~h} /$ day from a general media use scale (Pea et al., 2012).

Self-esteem. The Rosenberg Self-Esteem Scale (Rosenberg, 1965) was used to assess self-esteem on a 4-point scale with items such as "On the whole, I am satisfied with myself" and "I take a positive attitude toward myself." Select items in the 10-item scale were reversecoded and all averaged such that higher numbers indicated higher selfesteem. The measure had good internal consistency $(\alpha=0.92)$.

Depressive Symptoms. Depressive symptoms was assessed using the 20-item Center for Epidemiologic Studies Depression Scale (CES-D; Radloff, 1977). Participants reported on a 4-point scale how often they experienced depressive symptoms (e.g., "You were bothered by things that usually don't bother you," "You felt sad") in the past month $(\alpha=0.90)$.

Support. Perceived availability of instrumental, informational, and emotional support was assessed using a 12 -item scale of general social support developed for the National Institutes of Health $(\mathrm{NIH})$ PROMIS (Patient-Reported Outcomes Measurement Information System). Items (e.g., "I have someone to take me to the doctor if I need it," "I have someone to give me good advice about a crisis if I need it," and "I have someone to talk with when I have a bad day") were rated on a 5-point scale from 1 (never) to 5 (always) and had good internal consistency $(\alpha=0.96)$.

\section{Analytic Strategy}

As shown in Figure 1, acculturation strategies are derived from two dimensions or issues 1) maintaining offline ties and 2) preference for online connection. Therefore, issue 1 was assessed using the frequency of face-to-face (F2F) conversations and issue 2 was assessed using a frequency of digital media use for social connection. Four categories of integration (i.e., high F2F, high social media use), assimilation (i.e., low F2F, high social media use), separation (i.e., high F2F, low social media use), and marginalization (i.e., low F2F, low social media use) were created for each of the strategies based on mean-splits of measures for both dimensions of offline and online social connection. As a result, those in the low social media use group spent less than $2 \mathrm{~h}$ a day and those in the high social media use group spent more than $2 \mathrm{~h}$ a day emailing, messaging, and posting on social media, a cut-off recommended for practical importance in previous literature (Twenge, 2020).

Preliminary analyses suggests that participant age was negatively correlated with perceived support $(r=-0.31, p=0.016)$ and females were marginally more likely to spend time using social media $(t(58)=$ $-1.76, p=0.084)$. Additionally, ethnicity was coded into three groups based on sample sizes for each group: Latino, Asian American, and other ethnicities (e.g., African American, White, multi-ethnic). Participants from the other ethnicities group reported higher parent education $(F(2,57)=9.75, p<0.001)$ and higher depressive symptoms than Latino participants $(F(2,57)=3.99, p=0.024)$. However, there were no ethnic differences in in-person or online connection $(F s(2,56-57)=0.197,0.09$, ps $=0.822,0.912)$, self-esteem $(F(2,57)=0.21, p=0.812)$, or social support $(F(2,57)=1.63, p=$ $0.204)$. Given preliminary differences by age, gender, ethnicity, and socioeconomic status, these covariates were entered in ANCOVA models. Additionally, to account for the multiple ways participant identities and acculturation processes may overlap, years in the

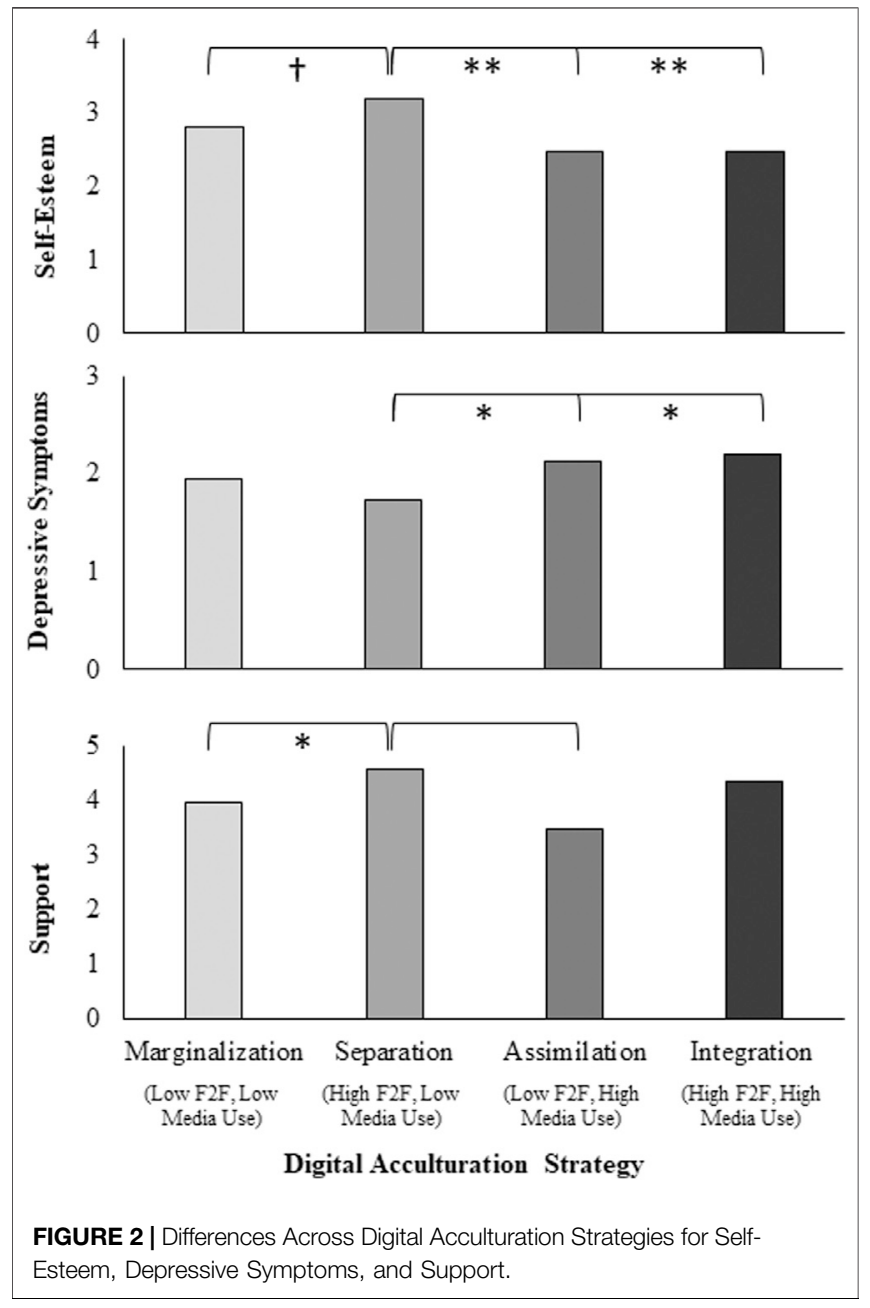

United States was included as a covariate. However, in the full models, gender, age, and parent education were not significant. Therefore, to conserve power, only ethnicity and years in the United States. were controlled for as covariates in final analyses. Furthermore, participants were part of a larger study that included an experimental manipulation in which they wrote a supportive letter to a friend, family member, or about their day. Experimental condition was controlled for in follow-up analyses and removed if it did not change the final results.

\section{RESULTS}

\section{Psychological Well-Being}

To test H1, an ANCOVA controlling for ethnicity and years in the United States assessed the effect of digital acculturation strategies on psychological well-being (i.e., self-esteem, depressive symptoms). There was a main effect of digital media acculturation strategy on self-esteem, $F(3,51)=3.50$, $p=0.022, \eta^{2}=0.17$. As shown in Figure 2, follow-up t-tests indicate partial support of $\mathrm{H} 1$ and $\mathrm{H} 3$ in that those in the separated group (high F2F, low social media use) had 
significantly higher self-esteem than the assimilated group (low F2F, high social media use; $t(26)=2.97, p=0.006$ ) and marginally higher than the marginalized group (low F2F, low social media use; $t(38)=1.82, p=0.077)$. However, contrary to hypotheses, they also reported higher self-esteem than the integrated group (high F2F, high social media use; $t(29)=3.55, p=0.001$ ).

A similar ANCOVA controlling for ethnicity and years in the United States showed a marginal main effect of digital acculturation strategy on depressive symptoms, $F(3,51)=2.67, p=0.057, \eta^{2}=0.14$. Follow-up t-tests indicate that the separated group reported significantly lower depressive symptoms than the assimilated group $(t(26)=-2.31, p=0.029)$. However, similar to earlier findings, they also reported lower depressive symptoms relative to the integrated group $(t(29)=-2.55, p=0.017)$.

\section{Social Well-Being}

Lastly, an ANCOVA controlling for ethnicity and years in the United States showed a significant main effect of digital acculturation strategy on perceived social support, $F(3,51)=$ 4.64, $p=0.006, \eta^{2}=0.21$. In support of $\mathrm{H} 2$ and $\mathrm{H} 3$, follow-up t-tests indicate that the separated group reported significantly higher social support than the marginalized $(t(38)=2.48, p=$ $0.018)$ and assimilated groups $(t(26)=3.10, p=0.005)$. People in the integrated group were not significantly different to the separated group in social support $(t(29)=1.13, p=0.269)$.

\section{DISCUSSION}

There was partial support for the hypothesis that people who maintained offline social connection reported higher mental wellbeing (i.e., higher self-esteem and lower depressive symptoms) relative to people who reported low offline social connection (H1). However, this was sustained primarily by people who adopted a separation strategy (i.e., high F2F conversations, low social media use) and particularly relative to those who adopted assimilation strategies (i.e., low F2F, high social media use). Again, partially consistent with $\mathrm{H} 2$, people who maintained high offline social connection, particularly those who reported high F2F conversations and low social media use (i.e., separated group), reported higher social wellbeing or social resources in the form of social support than those in the marginalization and assimilation groups.

Contrary to the acculturation literature, those who adopted an integration strategy (i.e., high F2F, high social media use) often fared similar to those in the assimilation group in terms of self-esteem and depressive symptoms. Although the integration group reported significantly poorer psychological well-being relative to the separation group, people in both groups perceived high levels of social support. It could be that people high in need (e.g., experiencing high stress due to a crisis) are more likely to seek out a wider range of social support (Uchino, 2006), as in those adopting an integration strategy. However, the results can also suggest that large social networks, especially those that consist of weak ties, can represent more of a burden than a blessing, reducing cognitive resources and increasing information overload (Chan, 2015).

Though the separation strategy for low social media use may be protective, it may also have negative implications for mental health as prior research suggests that not actively engaging or interacting with others online is associated with lower self-esteem (i.e., "lurking"; Liu and Baumeister, 2016) and poorer psychosocial functioning (Przybylski et al., 2020). It could be that people who experience rejection or negative experiences online are more likely to reject it in a form of "reactive identification" and adopt a separation strategy to protect against or promote mental well-being (Berry and Hou, 2016). Given the correlational design, these explanations are post hoc and speculative. More information is needed to fully conceptualize these groups (e.g., have those who adopt marginalization or separation strategies experienced more negative experiences or issues of access online?).

Finally, there was support for $\mathrm{H} 3$ in which participants using a strategy that most displaces in-person social interaction (i.e., high social media use combined with low F2F among the assimilated group) exhibited the worst psychological and social well-being. Altogether, rather than establishing the benefits of seeking connection in both online and offline contexts, the results provide stronger support for the idea that high use of social media that detracts from in-person connection can come at a cost to cognitive and psychological capacities (Chan, 2015; Twenge, 2020). Although multimodal connection helps cultivate larger social networks, it may contain weaker social ties (e.g., acquaintances, former classmates) that are less protective than stronger ties (e.g., close family and friends). High social media use and low F2F frequency alone did not guarantee poorer outcomes; instead, the interplay between offline and online social connection highlighted the complexities of multiple modes of connection for psychological and social well-being. This is consistent with reviews of the literature that suggest null or small effects for screen time or time spent on social media alone on mental health (Yoon et al., 2019; Orben, 2020) and point, rather, to specific usage patterns (Liu and Baumeister, 2016).

Although age was not correlated with offline conversation frequency or social media use, it could be that people who are not active on social media (i.e., utilized a marginalization or separation strategy) are merely later-adopters of social media who will become more entrenched online with time or circumstances (e.g., pandemicrelated lockdown due to COVID-19). It could also be that people who have poorer self-esteem or health are more likely to self-select in or out of social media contexts given longitudinal studies strongly suggest a link from self-esteem to social media use rather than vice versa (Valkenburg et al., 2017). The cross-sectional and correlational nature of the current study limits our exploration of these alternative explanations or to establish causal links and future studies that assess how strategies change over time can identify generational trends. In addition to longitudinal methods, examining patterns of use among individuals at-risk or suffering from depression and other mental health disorders may shed light on these relationships (Orben, 2020).

The study was also underpowered due to small and unequal sample sizes across groups. The results of this study, with our diverse sample of mostly female, young adult college students, can not generalize to populations of older "digital immigrants" who may use social media to connect with existing and emotionally-close ties (Chan, 2015). Our preliminary analyses showing marginally higher female social media use is in line with prior research that suggests gender differences in 
motives and patterns of media use (e.g., Liu and Baumeister, 2016; Orben, 2020). Also, although there were no ethnic differences in social media use or F2F communication, Latino participants reported lower parent education levels, a proxy for socioeconomic status that can indicate reduced access to either online or offline communication (e.g., participants who own multiple devices, such as a smartphone and laptop, are likely to have more opportunities for social media or multitasking during F2F conversations). Given that patterns of use and access are not universal or ubiquitous, future research should examine how factors like age, gender, ethnicity, socioeconomic status, and even region can determine strategies for adopting media and how those strategies affect well-being.

Additionally, there are several limitations to the measures in the current study. The social media use item included email, which may be different from other forms of social media use, particularly for this young adult population who are more likely to use it for school or work where there are weaker emotional ties (Chan, 2015). Although the outcome measures in the current study were selected to parallel previous research that has linked acculturation and psychosocial wellbeing (e.g., Berry, 2017), we did not measure other forms of relating to different cultural contexts (e.g., identity, language, norms) or acculturative stress (i.e., stress associated with adjusting to new cultural environments) and future studies should consider how psychological, social, or cognitive stress specific to acculturating to cultural contexts online affect well-being. Finally, the measures were self-report and may be prone to recall bias. Capturing media use with daily diary, ecological momentary assessment (EMA), or objective measures that track feature use may reduce bias in reporting (e.g., Jensen et al., 2019; Orben, 2020).

Lastly, there are differences in how the acculturation model, that has focused on immigrant-receiving countries and permanent resettlement processes rather than temporary sojourners (e.g., international students, diplomats, guest workers), and the proposed digital acculturation model, which can involve more regular and recurrent interchange between online and offline environments, can be applied to cultural and psychological development. Given the interactivity of digital media, users can shape their online environments as well as be shaped by them (Hong and Na, 2017). Relatedly, although years in the United States (a potential indicator of other occurring acculturation processes) were accounted for in the current study, the intersectionality of participant identities can interact in ways that shape mental well-being. For example, in the current study, individuals from Latino backgrounds reported lower levels of depressive symptoms compared to individuals from other ethnicities. Disparities in media use by sociodemographic factors may shape use and how it affects individuals from different immigrant and cultural backgrounds (e.g., Liu and Baumeister, 2016; Guan et al., 2017).Future research should further unpack and test different mechanisms in explaining how social media use affects the mental, social, and psychological health and development for incoming generations of immigrants and digital denizens (e.g., multiple sources of acculturative stress, specific social media activities and cultural self-presentation, health behaviors like sleep).

Despite limitations, many of the results showed medium to large effect sizes, suggesting the utility of drawing from frameworks in acculturation and digital media research. Recent research suggests that social displacement may occur at different levels of experience (e.g., at the daily, within-person level vs. between-person level; Verduyn et al., 2020). The results show concerning implications for people who spend more time online, perhaps at the expense of in-person socialization and particularly if these trends are part of generation-level shifts in cultural norms (Twenge, 2020). However, the findings may also be reflective of on-going acculturation or support-seeking processes rather than purely due to problematic or intractable offline traits.

The recent COVID-19 pandemic has highlighted the negative consequences of reduced in-person interaction and the transition to virtual on mental well-being, particularly for adolescents (Guessoum et al., 2020; Magson et al., 2021). However, in the face of limited faceto-face connection, social media has provided important means to communicate and alleviate anxieties (Magson et al., 2021). The American Psychological Association (APA) even recommends individuals stay connected virtually to promote mental well-being while face-to-face interactions are limited during the pandemic. Although this research suggests that social displacement can leave individuals vulnerable to poorer mental well-being, the current pandemic has highlighted how modern communication technologies can still provide opportunities for connection that may mitigate some of the negative consequences of lack of inperson connection as has been suggested in the literature (Jensen et al., 2019). Given the increasing prevalence of digital media and technology, especially in light of the COVID-19 pandemic, the current study highlights the need to successfully navigate between multiple social contexts and how some groups may be more vulnerable to the loss of supportive social ties critical to mental and physical health during periods of digital, cultural, and social transformation.

\section{DATA AVAILABILITY STATEMENT}

The data used in this article can be made available from the author upon reasonable request.

\section{ETHICS STATEMENT}

All procedures involving human participants were reviewed and approved by California State University, Northridge IRB \#1516221-d. The participants provided their written informed consent to participate in this study.

\section{AUTHOR CONTRIBUTIONS}

The author confirms being the sole contributor of this work and has approved it for publication.

\section{FUNDING}

This study was supported by the by the American Psychological Association (APA) Promoting Psychological Research and Training on Health Disparities Issues at Ethnic Minority Serving Institutions (ProDIGs) Grant. 


\section{REFERENCES}

Berry, J. W. (2005). Acculturation: Living Successfully in Two Cultures. Int. J. intercultural relations. 29 (6), 697-712. doi:10.1016/j.ijintrel.2005.07.013

Berry, J. W. (2017). "Theories and Models of Acculturation," in Oxford Library of Psychology. The Oxford Handbook of Acculturation and Health. Editors S. J. Schwartz and J. Unger (Oxford University Press), 15-28.

Berry, J. W., and Hou, F. (2016). Immigrant Acculturation and Wellbeing in Canada. Can. Psychology/Psychologie Canadienne. 57 (4), 254-264. doi:10.1037/cap0000064

Chan, M. (2015). Multimodal Connectedness and Quality of Life: Examining the Influences of Technology Adoption and Interpersonal Communication on Well-Being across the Life Span. J. Comput-mediat Comm. 20 (1), 3-18. doi: $10.1111 /$ jcc 4.12089

Guan, S.-S. A., Chiang, J. J., Sherman, L. E., Nguyen, J., Tsui, Y., and Robles, T. F. (2017). Culture Moderates the Effect of Social Support across Communication Contexts in Young Adult Women in the United States. Comput. Hum. Behav. 75, 775-784. doi:10.1016/j.chb.2017.05.048

Guessoum, S. B., Lachal, J., Radjack, R., Carretier, E., Minassian, S., Benoit, L., et al. (2020). Adolescent Psychiatric Disorders during the COVID-19 Pandemic and Lockdown. Psychiatry Res. 291, 113264. doi:10.1016/j.psychres.2020.113264

Hong, S., and Na, J. (2017). How Facebook Is Perceived and Used by People across Cultures. Soc. Psychol. Personal. Sci. 9 (4), 435-443. doi:10.1177/ 1948550617711227

Jensen, M., George, M. J., Russell, M. R., and Odgers, C. L. (2019). Young Adolescents' Digital Technology Use and Mental Health Symptoms: Little Evidence of Longitudinal or Daily Linkages. Clin. Psychol. Sci. 7 (6), 1416-1433. doi:10.1177/2167702619859336

Liu, D., and Baumeister, R. F. (2016). Social Networking Online and Personality of self-worth: A Meta-Analysis. J. Res. Personal. 64, 79-89. doi:10.1016/ j.jrp.2016.06.024

Magson, N. R., Freeman, J. Y. A., Rapee, R. M., Richardson, C. E., Oar, E. L., and Fardouly, J. (2021). Risk and Protective Factors for Prospective Changes in Adolescent Mental Health during the COVID-19 Pandemic. J. Youth Adolescence. 50 (1), 44-57. doi:10.1007/s10964-020-01332-9

Orben, A. (2020). Teenagers, Screens and Social media: a Narrative Review of Reviews and Key Studies. Soc. Psychiatry Psychiatr. Epidemiol. 55 (4), 407-414. doi:10.1007/s00127-019-01825-4

Pea, R., Nass, C., Meheula, L., Rance, M., Kumar, A., Bamford, H., et al. (2012). Media Use, Face-To-Face Communication, media Multitasking, and Social Well-Being Among 8- to 12-Year-Old Girls. Developmental Psychol. 48 (2), 327-336. doi:10.1037/a0027030
Prensky, M. (2001). Digital Natives, Digital Immigrants. On the Horizon. 9 (6), 1. doi:10.1108/10748120110424843

Przybylski, A. K., Orben, A., and Weinstein, N. (2020). How Much Is Too Much? Examining the Relationship between Digital Screen Engagement and Psychosocial Functioning in a Confirmatory Cohort Study. J. Am. Acad. Child Adolesc. Psychiatry 59 (9), 1080-1088. doi:10.1016/j.jaac.2019.06.017

Radloff, L. S. (1977). The CES-D Scale. Appl. Psychol. Meas. 1 (3), 385-401. doi:10.1177/014662167700100306

Rosenberg, M. (1965). Society and the Adolescent Self-Image. Princeton, NJ: Princeton University Press.

Song, H., Zmyslinski-Seelig, A., Kim, J., Drent, A., Victor, A., Omori, K., et al. (2014). Does Facebook Make You Lonely? A Meta Analysis. Comput. Hum. Behav. 36, 446-452. doi:10.1016/j.chb.2014.04.011

Twenge, J. M. (2020). Increases in Depression, Self-Harm, and Suicide Among U.S. Adolescents after 2012 and Links to Technology Use: Possible Mechanisms. Psychiatr. Res. Clin. Pract. 2 (1), 19-25. doi:10.1176/appi.prcp.20190015

Uchino, B. N. (2006). Social Support and Health: a Review of Physiological Processes Potentially Underlying Links to Disease Outcomes. J. Behav. Med. 29 (4), 377-387. doi:10.1007/s10865-006-9056-5

Unger, J. B., Gallaher, P., Shakib, S., Ritt-Olson, A., Palmer, P. H., and Johnson, C. A. (2002). The AHIMSA Acculturation Scale:. The J. Early Adolescence. 22 (3), 225-251. doi:10.1177/02731602022003001

Valkenburg, P. M., Koutamanis, M., and Vossen, H. G. M. (2017). The Concurrent and Longitudinal Relationships between Adolescents' Use of Social Network Sites and Their Social Self-Esteem. Comput. Hum. Behav. 76, 35-41. doi:10.1016/j.chb.2017.07.008

Verduyn, P., Schulte-Strathaus, J. C., Kross, E., and Hülsheger, U. R. (2020). When Do Smartphones Displace Face-To-Face Interactions and what to Do about it? Comput. Hum. Behav. 114, 106550. doi:10.1016/j.chb.2020.106550

Yoon, S., Kleinman, M., Mertz, J., and Brannick, M. (2019). Is Social Network Site Usage Related to Depression? A Meta-Analysis of Facebook-Depression Relations. J. Affective Disord. 248, 65-72. doi:10.1016/j.jad.2019.01.026

Conflict of Interest: The author declares that the research was conducted in the absence of any commercial or financial relationships that could be construed as a potential conflict of interest.

Copyright $(2021$ Guan. This is an open-access article distributed under the terms of the Creative Commons Attribution License (CC BY). The use, distribution or reproduction in other forums is permitted, provided the original author(s) and the copyright owner(s) are credited and that the original publication in this journal is cited, in accordance with accepted academic practice. No use, distribution or reproduction is permitted which does not comply with these terms. 